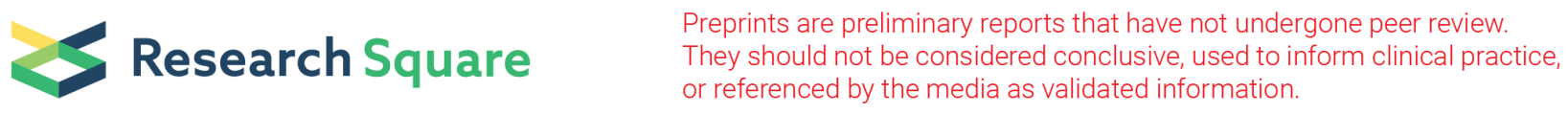

\title{
Simultaneous Detection of Pathogens and Tumors in Patients With Suspected Infections by Next-Generation Sequencing
}

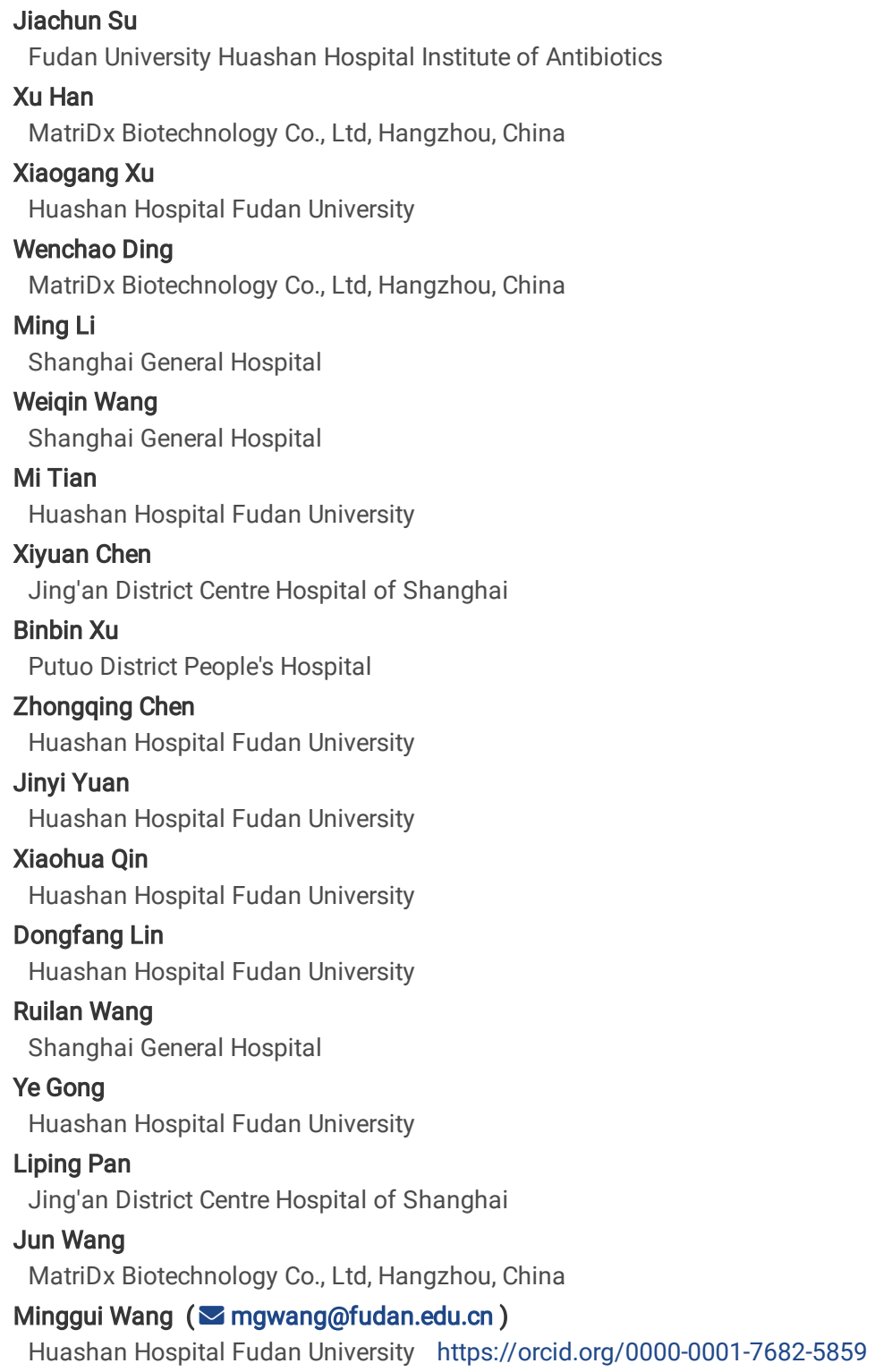

\section{Research Article}

Keywords: Onco-mNGS, Copy number variation, Pathogen, Infection, Tumor

Posted Date: April 14th, 2021

DOI: https://doi.org/10.21203/rs.3.rs-325171/v1

License: (c) (i) This work is licensed under a Creative Commons Attribution 4.0 International License. Read Full License 


\section{Abstract}

Background: Differential diagnosis of patients with suspected infections is particularly difficult, but necessary for prompt diagnosis and rational use of antibiotics. A substantial proportion of these patients have non-infectious diseases that include malignant tumors. Metagenomic next-generation sequencing (mNGS) technologies are used with increasing frequency to aid clinical diagnosis of patients with suspected infections.

Methods: Based upon mNGS technologies and chromosomal copy number variation (CNV) analysis on abundant human genome, a new workflow named Onco-mNGS was established to simultaneously detect pathogens and malignant tumors in patients with suspected infections.

Results: Of 140 patients screened by Onco-mNGS testing at four hospitals in Shanghai, 115 patients were diagnosed with infections; 17 had obvious abnormal CNV signals indicating malignant tumors that were confirmed clinically. The sensitivity and specificity of mNGS testing for diagnosis of a clinically relevant infection was $53.0 \%$ (61/115) and 60\% (15/25), respectively, vs $20.9 \%(24 / 115)$ and $96.0 \%(24 / 25)$, respectively, for conventional microbiological testing (both $P<0.01)$. Klebsiella pneumoniae was the most common pathogen detected by mNGS, followed by $E$. coli and viruses. The chromosomal abnormalities of the 17 cases included genome-wide variations and local variations of a certain chromosome. Five of 17 patients had a final confirmed with malignant tumors, including three lung adenocarcinomas and two hematological tumors; one patient was highly suspected to have lymphoma; and 11 patients had a prior history of malignant tumor.

Conclusions: This preliminary study demonstrates the feasibility and clinical value of using Onco-mNGS to simultaneously search for potential pathogens and malignant tumors in patients with suspected infections.

\section{Background}

It is often difficult to distinguish between malignant tumors and infectious diseases based upon clinical manifestations. Symptoms such as fever indicative of infections can occur in patients with malignant tumors. Indeed, the differential diagnosis of a "fever of unknown origin" is the most wide-ranging diagnosis in medicine[1]. One study, which summarized the results collected from over 1,000 patients with fever of unknown origin, indicated that the spectrum of diseases included infection (28\%), inflammatory disease (21\%), malignancy (17\%) and no diagnosis (19\%) [2]. Hematologic tumors, especially lymphoma, are the most common malignant tumors that induce fevers of unknown origin [3]. Bleeker-Rovers and co-workers reported that $58 \%$ and $5 \%$ of patients with suspected focal infections or inflammation had final diagnoses of infections and malignant tumors, respectively [4]. It is well-appreciated throughout medicine that the inability to obtain a timely and accurate diagnosis frequently results in unnecessary antimicrobial treatments, high healthcare costs, and poor prognoses. Therefore, the differential diagnosis of patients with suspected infection is particularly necessary.

Metagenomic next-generation sequencing (mNGS), which has been used in clinical practice for several years, represents an innovative strategy for detecting potential pathogens including bacteria, viruses, fungi, and parasites [5-7]. Indeed, studies published to date that explored the clinical applications of mNGS focused primarily on the specific sources of infection, e.g., focal infections, meningitis and pneumonia [6, 8-10]. Recent mNGS studies demonstrated that the nucleic acid yields from pathogens account for only 0.00001 to $1-2 \%$ of the total reads; human sequences (homo reads) represent $\geq 90 \%$ [ $11-13$ ]. It is possible to obtain valuable information from this large homo reads database. Chromosome instability, defined as a defect that involves chromosomal loss or rearrangement during tumorigenesis, is a hallmark of cancer [14]. NGS-based methods used to analyze chromosomal copy number variation (CNV) employ experimental procedures very similar to those used for mNGS.

Considering CNV analysis is already used to assist the diagnosis of genetic diseases and cancer $[15,16]$, it is conceivable that mNGS homo reads data can be used for tumor detection. Here, a new analysis workflow (Onco-mNGS) is proposed for using homo reads data to search for indications of malignant tumors. The results of this multicenter study show that Onco-mNGS not only improves diagnosis of infection, but also provides evidence of malignant tumors in patients with suspected infections.

\section{Methods}

\section{Study participants}

This was a multicenter, prospective case study in which patients were enrolled between Jul 2019 and Jan 2020 upon diagnosis of a suspected infection.

Demographic and clinical data collected for each patient included: sex, age, underlying disease, admission to an intensive care unit (ICU), routine blood examination, clinical microbiological results (cultures for bacteria, acid-fast bacilli and fungi), and pathological reports (pathological types, immunohistochemical markers including CD20, Ki67). Physicians conducted other conventional tests, e.g., tests for EBV and CMV, according to their clinical judgment. Peripheral blood $(\sim 5 \mathrm{~mL})$ and clinical fluid samples were collected in Cell-Free DNA Collection Tubes (Cat. CAB0305-100, Lakebio Corp. Hefei). All samples were sequenced within two days of collection.

\section{NGS sequencing}

All samples were subjected to DNA extraction, library preparation, and next-generation sequencing (NGS). DNA extraction and library preparation were conducted on the NGS Automatic Library Preparation System (Cat. MAR002, MatriDx Biotech Corp. Hangzhou). Reagents included: Nucleic Acid Extraction Kit (Cat. MD013, MatriDx Biotech Corp. Hangzhou), Cell-free DNA Library Preparation Kit (blood samples) (Cat. MD007, MatriDx Biotech Corp. Hangzhou) and Total DNA Library Preparation Kit (other samples) (Cat. MD001T, MatriDx Biotech Corp. Hangzhou). Libraries were pooled then sequenced on an Illumina NextSeq500 system using a 75-cycle sequencing kit. A total of 10-20 million reads were obtained for each sample.

\section{mNGS analysis}


First, human sequences were excluded in mNGS analysis by aligning all sequence reads with the human reference genome (hy19). The remaining reads were aligned with the NCBI reference sequence database using Kraken2 and bowtie2 $[17,18]$. Then, an in-house pipeline was used to process the result of alignment to determine the microbial composition of the sample.

Finally, potential pathogens were selected from the results of analysis according to the clinical phenotype using machine-learning models; these data were reviewed by senior clinicians.

\section{Chromosomal copy number variation (CNV) analysis}

Sequencing reads were aligned with the human reference genome (hg19) and only unique, mapped reads were selected for subsequent analysis. The reference genome was segmented into continuous windows of fixed length to calculate the read depth of each window.

The number of total unique reads was calculated by adding the window read depth on autosomes and normalized to $3,000,000$ yielding a normalization coefficient. The read depth of each window was then multiplied by this coefficient to eliminate the effect of sequencing depth differences between samples.

Copy number ratios were obtained by dividing the read depth of each window by the average read depth in the reference dataset. Afterwards, the fused lasso method (a generalization of the lasso penalty for sequential signal smoothing with sparsity) was applied to log2-transformed copy number ratios. Smoothed adjacent windows with similar ratios were merged into segments with chromosome positions and average ratios annotated. The copy number of each segment was calculated according to the average ratio and normal copy number of the corresponding chromosomes and then compared with preset thresholds to validate a CNV.

\section{Statistics}

Data analyses were performed using SPSS 22.0 software. Baseline characteristics were summarized using descriptive statistics. Categorical variables were compared using the Chi-square test. All $P$ values were two-sided, with a $P$ value $<0.05$ considered statistically significant.

\section{Results}

\section{Patient characteristics and pathogen detection}

One hundred and forty patients with a $67.9 \%$ male to female ratio and a mean age of 55.8 years were enrolled at four hospitals. Fever or hypothermia was the most frequent symptom observed (77.9\%), followed by change in mental status (22.1\%), focal pain or dysfunction (18.6\%), and cough (17.1\%). Most patients $(92.8 \%)$ came from Intensive Care Units or Departments of Infectious Disease. A total of $92.9 \%$ of patients received antimicrobial treatment in the two weeks prior to mNGS examination. The following samples were collected for mNGS testing from patients recruited for the study: peripheral blood (110 patients), bronchoalveolar lavage fluid (BALF) (24 patients), cerebrospinal fluid (CSF) (32 patients), and other types, e.g., sputum, pleural fluid, ascites, and pericardial effusion (29 patients) (Fig. 1A).

Seventy-one patients have detected a potential pathogen (environmental microbes which considered as contaminants were filtered), of which bacteria, fungi and virus accounted for $65 \%, 10 \%$ and $10 \%$, respectively. In addition, $15 \%$ of patients may have combined infection (Fig. 1B).

A total of 115 out of 140 patients received a final diagnosis of infection; pneumonia was the most common $(66.1 \%, 76 / 115)$, followed by infections of the central nervous system $(19.3 \%, 26 / 115)$ and bloodstream $(13.9 \%, 16 / 115)$. Twenty-five patients $(21.7 \%)$ had more than one source of infection. The positive mNGS tests of 61 patients were assessed to be clinically relevant. The sensitivity and specificity of mNGS testing for diagnosis of a clinically relevant infection was 53.0\% (61/115) and 60\% (15/25), respectively, vs $20.9 \%(24 / 115)$ and $96.0 \%(24 / 25)$, respectively, for conventional microbiological testing (both $P<0.01)$. Klebsiella pneumoniae was the most common pathogen detected by mNGS, followed by $E$. coli and viruses.

\section{Integrate copy number variation analysis with mNGS}

The main objective of the current study was to explore the possibility of using mNGS to detect malignant tumors. Inasmuch as the experimental approach is consistent with current methods of mNGS library preparation, a new analysis workflow (Onco-mNGS) is proposed here for using homo reads data to search for evidence of malignant tumors (Fig. 2A). A process was established based upon the read counting strategy.

This process was tested first using known pathologic samples. Standard diploid samples with no chromosomal deletions or duplications were obtained from healthy individuals (Fig. 2B). In contrast, dramatic chromosome disorders were observed in tumor tissues obtained from two colorectal cancer patients. Considering the proportion of tumor cells in the samples could be low, the sensitivity of the method was further examined by preparing and testing a variety of samples with different tumor to normal cell ratios (Supplementary Fig. S1). Chromosome abnormalities were readily detected at ratios of 5-20\% supporting the overall concept of using mNGS technologies to identify malignant tumors. These results support the feasibility of integrating CNV analysis with mNGS to detect tumors.

\section{Analysis of homo reads for tumor clues}

Next, CNV analysis was performed on each study sample. The homo read ratios were calculated for the three major sample types: peripheral blood, BALF and CSF. The average ratio of homo reads for each sample type was > 95\% (plasma: 96.26\%; BALF: 96.03\%; CSF: 95.84\%) (Fig. 3A). After excluding microbial sequences, $\geq 8$ million homo reads were obtained for each sample, enough to conduct CNV analysis. Seventeen samples with significant chromosomal disorders were identified. Among these, ten were peripheral blood and seven were body fluid samples (three BALF, two pleural fluid, and two CSF). Abnormal CNV signals were observed in both peripheral blood and BALF samples obtained from one patient (\#12). 
The following chromosome abnormalities were observed in these 17 patients: 1) genome-wide multiple chromosome duplication and deletion in 10 patients (\#s 1, 2, 3, 4, 5, 10, 11, 13, 16 and 17); 2) arm level duplication and deletion in four patients (\#s 6, 8, 9, 14); 3 ) loss of chromosome $Y$ in two patients (\#s 7 and 12); and 4) chromosome aneuploidy in one patient (\# 15) (Fig. 3B and Supplementary Fig. S2). The genome-wide chromosome abnormality was observed to be the most type of CNV variation (Fig. 3D).

\section{Clinical validation of Onco-mNGS}

Ten peripheral blood samples were obtained from these 17 patients. Additionally, tumor-like signals were found in body fluid samples (three BALF, two pleural fluid, and two CSF). Six patients with obvious, abnormal CNV signals had no recorded history of malignant tumors prior to Onco-mNGS. Malignant tumors were subsequently confirmed in five patients (\#s 1-5). Three lung adenocarcinomas and two hematological tumors were determined by pathologic examination of tissues obtained by bone marrow biopsy, bone biopsy or surgery after the results of Onco-mNGS analysis were reported to the attending physicians (Fig. 3C and Table 1). The remaining patient (\#6) was suspected of having central nerve system lymphoma, but refused to undergo a biopsy prior to death (Table 1 and Fig. 3).

Table 1

Clinical characteristics of patients with CNV changes and no history of malignancy before mNGS testing

\begin{tabular}{|c|c|c|c|c|c|c|c|c|c|c|c|}
\hline \multirow[t]{2}{*}{ No. } & \multirow{2}{*}{$\begin{array}{l}\text { Sex, } \\
\text { year }\end{array}$} & \multirow{2}{*}{$\begin{array}{l}\text { History of } \\
\text { malignancy }\end{array}$} & \multirow{2}{*}{$\begin{array}{l}\text { Diagnosis } \\
\text { of } \\
\text { admission }\end{array}$} & \multirow{2}{*}{$\begin{array}{l}\text { Diagnosis } \\
\text { of } \\
\text { discharge }\end{array}$} & \multirow[t]{2}{*}{ Symptom } & \multirow{2}{*}{$\begin{array}{l}\text { Prior } \\
\text { antimicrobial } \\
\text { agents use }\end{array}$} & \multicolumn{3}{|l|}{ Onco-mNGS } & \multicolumn{2}{|c|}{ Conventional m€ } \\
\hline & & & & & & & $\begin{array}{l}\text { Pathogen } \\
\text { detected }\end{array}$ & CNVs & $\begin{array}{l}\text { Days to } \\
\text { diagnosis }^{a}\end{array}$ & $\begin{array}{l}\text { Culture } \\
\text { result }\end{array}$ & $\begin{array}{l}\text { Patho } \\
\text { detect }\end{array}$ \\
\hline 1 & $\begin{array}{l}\text { Female, } \\
58\end{array}$ & None & $\begin{array}{l}\text { Thoracic } \\
\text { vertebrae } \\
\text { lesion }\end{array}$ & $\begin{array}{l}\text { Diffuse } \\
\text { large B cell } \\
\text { lymphoma }\end{array}$ & $\begin{array}{l}\text { Chest } \\
\text { and back } \\
\text { pain }\end{array}$ & $\begin{array}{l}\text { Vancomycin; } \\
\text { Fosfomycin }\end{array}$ & None & $\begin{array}{l}\text { Multiple } \\
\text { Chrs }^{\text {b }} \\
\text { Del/Dup }\end{array}$ & 14 days & None & $\begin{array}{l}\text { Bone ! } \\
\text { diffus } \\
\text { cell lyr }\end{array}$ \\
\hline 2 & $\begin{array}{l}\text { Male, } \\
67\end{array}$ & None & $\begin{array}{l}\text { Liver } \\
\text { abscess }\end{array}$ & $\begin{array}{l}\text { Lung } \\
\text { cancer; } \\
\text { Liver } \\
\text { Occupation; } \\
\text { Pneumonia }\end{array}$ & $\begin{array}{l}\text { Fever; } \\
\text { Change } \\
\text { in metal } \\
\text { status }\end{array}$ & $\begin{array}{l}\text { Meropenem; } \\
\text { Ciprofloxacin; } \\
\text { Linezolid; }\end{array}$ & None & $\begin{array}{l}\text { Chr2 } \\
\text { Dup }\end{array}$ & 90 days & None & $\begin{array}{l}\text { Broncl } \\
\text { neoplá } \\
\text { superi } \\
\text { segme } \\
\text { broncl } \\
\text { Patho } \\
\text { adeno }\end{array}$ \\
\hline 3 & $\begin{array}{l}\text { Male, } \\
23\end{array}$ & None & $\begin{array}{l}\text { Fever of } \\
\text { unknown } \\
\text { origin }\end{array}$ & $\begin{array}{l}\text { NK T cell } \\
\text { lymphoma }\end{array}$ & $\begin{array}{l}\text { Fever; } \\
\text { Vomit }\end{array}$ & $\begin{array}{l}\text { Meropenem; } \\
\text { Doxycycline; } \\
\text { Vancomycin; } \\
\text { Cefoperazone; } \\
\text { Sulbactam }\end{array}$ & $\begin{array}{l}\text { Blood: Bacillus } \\
\text { thuringiensis }\end{array}$ & $\begin{array}{l}\text { Multiple } \\
\text { Chrs } \\
\text { Del/Dup }\end{array}$ & 6 days & None & $\begin{array}{l}\text { Bone I } \\
\text { smear } \\
\text { lymph }\end{array}$ \\
\hline 4 & $\begin{array}{l}\text { Female, } \\
71\end{array}$ & None & Lung lesion & $\begin{array}{l}\text { Lung } \\
\text { cancer }\end{array}$ & $\begin{array}{l}\text { Cough; } \\
\text { Sputum }\end{array}$ & None & None & $\begin{array}{l}\text { Multiple } \\
\text { Chrs } \\
\text { Del/Dup }\end{array}$ & 9 days & None & $\begin{array}{l}\text { Lung ! } \\
\text { adeno }\end{array}$ \\
\hline 5 & $\begin{array}{l}\text { Male, } \\
71\end{array}$ & None & $\begin{array}{l}\text { Pericardial } \\
\text { and pleural } \\
\text { effusion }\end{array}$ & $\begin{array}{l}\text { Lung } \\
\text { cancer }\end{array}$ & None & Moxifloxacin & None & $\begin{array}{l}\text { Multiple } \\
\text { Chrs } \\
\text { Del/Dup }\end{array}$ & 5 days & None & $\begin{array}{l}\text { Lung ! } \\
\text { adeno }\end{array}$ \\
\hline 6 & $\begin{array}{l}\text { Male, } \\
69\end{array}$ & None & $\begin{array}{l}\text { Encephalitis } \\
\text { possible }\end{array}$ & $\begin{array}{l}\text { Central } \\
\text { lymphoma } \\
\text { possible }\end{array}$ & $\begin{array}{l}\text { Fever; } \\
\text { Change } \\
\text { in metal } \\
\text { status }\end{array}$ & $\begin{array}{l}\text { Ceftriaxone; } \\
\text { Metronidazole }\end{array}$ & $\begin{array}{l}\text { CSF: } \\
\text { Elizabethkingia } \\
\text { anophelis }\end{array}$ & $\begin{array}{l}\text { Chr9q } \\
\text { Dup }\end{array}$ & 29 days & None & - \\
\hline
\end{tabular}

aDuration of time between the first day of hospitalization and the report of Onco-mNGS or pathological results.

bAbbreviations: Chr, chromosome; Dup, duplication; Del, deletion

The remaining 11 patients(\#s 7-17)with obvious abnormal CNV signals had a history of malignant tumors noted upon chart review (Supplementary Table S1 and Fig. S2). Hematologic tumors were the most frequently detected accounting for $63.6 \%$ of the malignant tumors. In this study, 30 of 140 patients in this study had a history of malignancy before Onco-mNGS analysis; 19 of these patients, however, were not found to have obvious abnormal CNV signals.

By combining the mNGS analyses with CNV analyses, 12 of the 17 patients with obvious abnormal CNV signals were found to have negative mNGS results, indicating malignant tumor may be the cause of infection-like symptoms such as fever (Fig. 3E).

\section{Case vignettes in which Onco-mNGS yielded a malignant diagnosis}

\section{Case 1}

Diffuse large B cell lymphoma. A 58-year-old female presented after 2 months with right chest and back pain with no fever (Fig. 4A). A CT scan revealed destruction of the 9th thoracic vertebra and the right appendix with paravertebral soft tissue shadow (Fig. 4B). Magnetic resonance imaging (MRI) revealed abnormal signals of the 9th thoracic vertebra and right arch with swelling of the surrounding soft tissue. Pathologic results of the bone biopsy indicated 
infection. The patient was treated empirically with vancomycin and fosfomycin (switched to linezolid due to nausea and vomiting) administered i.v. for 3 weeks. The patient developed paralysis of the lower extremities and difficulty urinating. Repeated MRI showed that the lesion had become significantly larger with spinal compression.

Onco-mNGS analysis failed to detect microorganisms in her peripheral blood, but revealed deletions and duplications of large segments in several chromosomes that generated a strong abnormal CNV-based tumor signal (Fig. 4C). This finding encouraged treating physicians to obtain a second bone biopsy; the result revealed diffuse large B cell lymphoma of the non-germinal center B cell type (Fig. 4D). The patient received R-CHOP chemotherapy afterwards and the symptoms were mildly relieved. The fluctuation in chromosomal variation was much smoother in the fourth, compared to previous, OncomNGS tests suggesting that the proportion of tumor cells had decreased.

\section{Case 2}

NK/T cell lymphoma. A 23-year-old male presented after 2 weeks of fever and vomiting. Upon admission, the patient exhibited a low platelet count (45·10 $/ \mathrm{L}$ ) and elevated levels of inflammatory markers (C-protein: $>199 \mathrm{mg} / \mathrm{L}$; procalcitonin: $13.92 \mathrm{ng} / \mathrm{mL}$ ). Tests for pathogens that included Epstein-Barr virus (EBV), cytomegalovirus (CMV), cryptococcus, tuberculosis, and parasites were negative; blood cultures for bacteria, acid-fast bacilli, and fungi were also negative. A PET-CT scan showed an enlarged spleen with increased uptake of FDG indicator. The patient underwent two diagnostic bone marrow punctures and a splenectomy; the results, however, did not support a definitive diagnosis. The patient was treated empirically with meropenem, vancomycin, doxycycline, gentamycin, and trimethoprim/sulfamethoxazole administered sequentially, but became worse with progressive hepatic deterioration (total bilirubin: 356.4 $\mu \mathrm{mol} / \mathrm{L})$. The results of a third bone marrow biopsy revealed NK/T cell lymphoma.

Onco-mNGS results showed disruptions in several chromosomes indicative of a tumor. This was confirmed by the results of a third bone marrow smear. Unfortunately, this information was not considered a priori by the attending physicians and the patient soon died of tumor progression. Although the diagnostic information provided by Onco-mNGS did not impact the clinical outcome of this patient directly, the case illustrates the potential benefits of this approach to patients with fever of unknown origin. It substantially shortens the time required for diagnosis and should eliminate unnecessary antimicrobial treatment.

\section{Case 3}

Lung adenocarcinoma. A 71-year-old female presented after 2 months of cough and sputum, but no fever. Her chest CT scan showed pneumonia; Mycoplasma pneumoniaespecific IgM antibody was positive. She was treated with cefuroxime and azithromycin administer i.v. for 6 days, then switched to oral moxifloxacin for 10 days. The patient did not improve in clinical or radiological presentation. Repeated, M. pneumoniae serum antibody was negative. Bronchoscopy was conducted, but tests for pathogens including bacteria, acid-fast bacteria, and fungi in BALF were negative. The patient received a pulmonary lobectomy and the pathological result indicated lung adenocarcinoma.

Tumor markers in this patient, i.e., CA199, CEA, CA125, CY211 and SCC, were in the normal range. A quick tumor diagnosis was overlooked since pneumonia was considered first. The Onco-mNGS result showed no microorganisms, but likely tumor signals. This finding supported the clinical decision to cease antimicrobial treatment and persuade the patient to receive invasive operations, which led to the final diagnosis.

\section{Discussion}

Suspected infections in patients may have alternate causes that include malignant tumors and autoimmune diseases. Timely identification of the specific cause is critical in treating these patients appropriately. mNGS was recently developed for infectious disease diagnostics and can theoretically detect all pathogens in clinical samples. Compared to traditional culture method, mNGS is noted for its enhanced capacity to detect fungi, viruses, anaerobes, and atypical pathogens (a unique ability among clinical approaches to diagnosing infections) [6, 7, 19]. Moreover, prior antibiotic treatment is less likely to affect the results of mNGS analysis [20]. While mNGS exhibits distinct advantages in diagnosing infectious diseases, its use in diagnosing non-infectious diseases has yet to be examined although the vast majority of data derives from the human genome. The current study demonstrates these data represent an extremely valuable diagnostic resource.

Previous studies reported abnormal CNVs suggestive of high genomic instability in cell-free DNA preparations obtained from patients with malignant tumors such as non-small cell lung and prostate cancers $[15,16]$. Genetic disease CNV and tumor-like CNV are very different. Genetic CNV usually entails duplication or deletion of large chromosomal segments; patients with large deletions and duplications or aneuploidies show serious genetic disease phenotypes at a very early age. CNVs caused by tumors appear as duplications or deletions of large fragments or entire chromosomes; copy number is generally not an integer. The types of anomalies differed among the 17 samples that exhibited significant chromosomal disorders in the current study. Some chromosomes showed global chaos while others showed local fluctuation. This may relate to tumor type and the ratio of tumor cells. Interestingly, loss of the $Y$ chromosome was observed in two cases, but these cases did not show any abnormalities related to the male sex chromosome. Rather, both patients had leukemia. Previous studies report that the $\mathrm{Y}$ chromosome is lost 15 to $80 \%$ of the time in cases involving a variety of cancer types [21-24]. It is anticipated that the utility of the homo read approach for exploiting mNGS data to support medical diagnoses will continue to increase. This increase will depend upon constantly up-dating the OncomNGS workflow to include new insights into CNVs (general, as well as disease-specific, manifestations) gathered from the medical literature.

Diagnosis of malignant tumors often requires multiple steps that can take a long period of time. Occasionally, invasive measures such as puncture or surgery are required. Understandably, the type of information obtained from homo read data using Onco-mNGS methodology does not provide a direct diagnosis; rather, it offers to contribute significantly to differential diagnoses. Importantly, combining CNV and mNGS analyses does not increase costs or require additional sampling. With the reduction of sequencing costs and the devolopment of technology, the current cost of mNGS has dropped to an acceptable level, 
and more and more clinical application scenarios have been demonstrated. In this study, with the help of the automated PCR-free library building platform (NGSmaster, MatriDx Biotech Corp. Hangzhou) [25], the turn-around time (TAT) can be shoten within 24 hours. Thus, utilization of Onco-mNGS alongside ongoing mNGS testing in hospitals should provide doctors a powerful new tool for making informed and quicker decisions during the diagnosis process.

In this study, 30 of 140 patients had a history of malignancy; only 11 of these patients expressed obvious, abnormal CNV signals. Considering the relatively short half-life of circulating free DNA (ranging from 16 minutes to 2.5 hours in peripheral blood) [26], the detection of chromosomal abnormalities in these patients suggest an active state of malignancy. It is reasonable to assume, therefore, that tumor cells exist and cause the clinical symptoms, e.g., fever, observed in these 11 patients. The accumulated clues that occur by considering both infection and malignant tumors as causative agents of disease will help formulate more effective treatment strategies. Previous studies also indicated that tumor CNV burden might foretell outcomes such as recurrence and survival [27]. It will be interesting to investigate Onco-mNGS as an approach to determine residual tumor or relapse in this patient group. In addition, infections are common complications occured in cancer patients, i.e., cancers are underlying diseases of infections. Onco-mNGS will be helpful to avoid delay of cancer diagnosis for patients with cancer and infection, who is usually diagnosed as infections by traditional culture method or mNGS.

The present study has several limitations. These include the relatively small number of patients with suspected infections that yielded the mNGS data examined herein, and the small number of malignant cases detected. Additionally, the real time disconnect between examination of the mNGS data and the treatment provided by attending physicians is far less than optimal. It is anticipated that the coordinated application of Onco-mNGS data and standard mNGS data analyses going forward will substantially shorten diagnostic time, and thereby increase the utility of this information.

\section{Conclusions}

Taken together, this study reported a new workflow named Onco-mNGS, based upon mNGS technologies and chromosomal CNV analysis, for simultaneously detection of pathogens and malignant tumors in patients with suspected infections. Onco-mNGS exhibited a significantly higher sensitivity rate for diagnosis of a clinically relevant infection than did traditional microbiological testing, and also enhanced the ability for early differentiation diagnosis of malignant tumor. Further efforts are needed to evaluate the clinical value of Onco-mNGS.

\section{Abbreviations}

mNGS: Metagenomic next-generation sequencing; CNV: Copy number variation; ICU: intensive care unit; NGS: Next-generation sequencing; BALF: Bronchoalveolar lavage fluid; CSF: Cerebrospinal fluid; EBV: Epstein-Barr virus; CMV: Cytomegalovirus

\section{Declarations}

\section{Ethics approval and consent to participate}

The study protocol was approved by the Ethics Committee of Huashan Hospital. Written informed consent was obtained from all study participants prior to their inclusion in the study.

\section{Consent for publication}

Not applicable.

\section{Availability of data and materials}

All data associated with this study are present in the main text or the Supplementary Materials. Individual participant data from the clinical study will not be shared.

\section{Competing interests}

The authors have declared that no conflict of interest exists.

\section{Funding}

This work was supported by the Natural Science Foundation of China (grant numbers 81991531, 81773785, and 81903673), Shanghai Municipal Science and Technology Commission (17411950704, 18411950600), and Shanghai Sailing Program (19YF1405400).

\section{Authors' contributions}

JS and XX collected and analyzed clinical data, and wrote the manuscript. XH conducted experiments, analyzed the data, and wrote the manuscript. WD conducted experiments and analyzed the data. ML, WW, MT, XC, BX, JY, XQ, DL, RW, YG, and LP included patients and collected clinical data. ZC had pathological examination. XX, JW, and MW designed the study and revised the manuscript.

\section{Acknowledgments}

The authors thank Dr. Stephen H. Gregory (Providence, RI, USA) for his help editing this manuscript.

\section{References}


1. Fusco FM, Pisapia R, Nardiello S, Cicala SD, Gaeta GB, Brancaccio G. Fever of unknown origin (FUO): which are the factors influencing the final diagnosis? A 2005-2015 systematic review. BMC Infect Dis. 2019;19:653.

2. Mourad O, Palda V, Detsky AS. A comprehensive evidence-based approach to fever of unknown origin. Arch Intern Med. 2003;163:545-51.

3. Zhou G, Zhou Y, Zhong C, Ye H, Liu Z, Liu Y, et al. Retrospective analysis of 1,641 cases of classic fever of unknown origin. Ann Transl Med. $2020 ; 8: 690$.

4. Bleeker-Rovers CP, de Kleijn EM, Corstens FH, van der Meer JW, Oyen WJ. Clinical value of FDG PET in patients with fever of unknown origin and patients suspected of focal infection or inflammation. Eur J Nucl Med Mol Imaging. 2004;31:29-37.

5. Chiu CY, Miller SA. Clinical metagenomics. Nat Rev Genet. 2019;20:341-55.

6. Wilson MR, Sample HA, Zorn KC, Arevalo S, Yu G, Neuhaus J, et al. Clinical metagenomic sequencing for diagnosis of meningitis and encephalitis. N Engl J Med. 2019;380:2327-40.

7. Miller S, Naccache SN, Samayoa E, Messacar K, Arevalo S, Federman S, et al. Laboratory validation of a clinical metagenomic sequencing assay for pathogen detection in cerebrospinal fluid. Genome Res. 2019;29:831-42.

8. Schlaberg R, Queen K, Simmon K, Tardif K, Stockmann C, Flygare S, et al. Viral pathogen detection by metagenomics and pan-viral group polymerase chain reaction in children with pneumonia lacking identifiable etiology. J Infect Dis. 2017;215:1407-15.

9. Zhang HC, Ai JW, Cui P, Zhu YM, Hong-Long W, Li YJ, et al. Incremental value of metagenomic next generation sequencing for the diagnosis of suspected focal infection in adults. J Infect. 2019;79:419-25.

10. Wilson MR, O'Donovan BD, Gelfand JM, Sample HA, Chow FC, Betjemann JP, et al. Chronic meningitis investigated via metagenomic next-generation sequencing. JAMA Neurol. 2018;75:947-55.

11. van Rijn AL, van Boheemen S, Sidorov I, Carbo EC, Pappas N, Mei H, et al. The respiratory virome and exacerbations in patients with chronic obstructive pulmonary disease. PloS one. 2019;14:e0223952.

12. Simner PJ, Miller S, Carroll KC. Understanding the promises and hurdles of metagenomic next-generation sequencing as a diagnostic tool for infectious diseases. Clin Infect Dis. 2018;66:778-88.

13. Salzberg SL, Breitwieser FP, Kumar A, Hao H, Burger P, Rodriguez FJ, et al. Next-generation sequencing in neuropathologic diagnosis of infections of the nervous system. Neurol Neuroimmunol Neuroinflamm. 2016;3:e251.

14. Hanahan D, Weinberg RA. Hallmarks of cancer: the next generation. Cell. 2011;144:646-74.

15. Zhang F, Gu W, Hurles ME, Lupski JR. Copy number variation in human health, disease, and evolution. Annu Rev Genomics Hum Genet. $2009 ; 10: 451-81$.

16. Heitzer E, Ulz P, Belic J, Gutschi S, Quehenberger F, Fischereder K, et al. Tumor-associated copy number changes in the circulation of patients with prostate cancer identified through whole-genome sequencing. Genome Med. 2013;5:30.

17. Wood DE, Lu J, Langmead B. Improved metagenomic analysis with Kraken 2. Genome Biol. 2019;20:257.

18. Langmead B, Salzberg SL. Fast gapped-read alignment with Bowtie 2. Nat Methods. 2012;9:357-59.

19. Blauwkamp TA, Thair S, Rosen MJ, Blair L, Lindner MS, Vilfan ID, et al. Analytical and clinical validation of a microbial cell-free DNA sequencing test for infectious disease. Nat Microbiol. 2019;4:663-74.

20. Miao Q, Ma Y, Wang Q, Pan J, Zhang Y, Jin W, et al. Microbiological diagnostic performance of metagenomic next-generation sequencing when applied to clinical practice. Clin Infect Dis. 2018;67:231-40.

21. Hunter S, Gramlich T, Abbott K, Varma V. Y chromosome loss in esophageal carcinoma: an in situ hybridization study. Genes Chromosomes Cancer. 1993;8:172-7.

22. Park SJ, Jeong SY, Kim HJ. Y chromosome loss and other genomic alterations in hepatocellular carcinoma cell lines analyzed by CGH and CGH array. Cancer Genet Cytogenet. 2006;166:56-64.

23. Bianchi NO. Y chromosome structural and functional changes in human malignant diseases. Mutat Res. 2009;682:21-7.

24. Duijf PH, Schultz N, Benezra R. Cancer cells preferentially lose small chromosomes. Int J Cancer. 2013;132:2316-26.

25. Luan Y, Hu H, Liu C, Chen B, Liu X, Xu Y, et al. A proof-of-concept study of an automated solution for clinical metagenomic next-generation sequencing. J Appl Microbiol. 2021. doi:10.1111/jam.15003.

26. Diehl F, Schmidt K, Choti MA, Romans K, Goodman S, Li M, et al. Circulating mutant DNA to assess tumor dynamics. Nat Med. 2008;14:985-90.

27. Hieronymus H, Murali R, Tin A, Yadav K, Abida W, Moller H, et al. Tumor copy number alteration burden is a pan-cancer prognostic factor associated with recurrence and death. eLife. 2018;7:e37294.

\section{Figures}

A

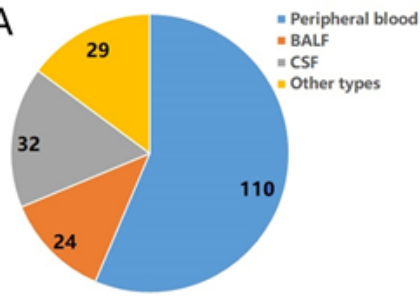

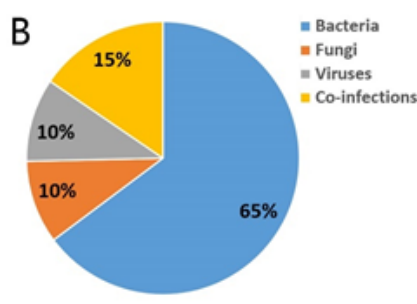




\section{Figure 1}

Summary of pathogens detected by mNGS. a Sample types tested in this study. Other types including sputum, pleural fluid, ascites, and pericardial effusion. b Distribution of infection types (bacteria, viruses, fungi and co-infection) in 140 patients.

A

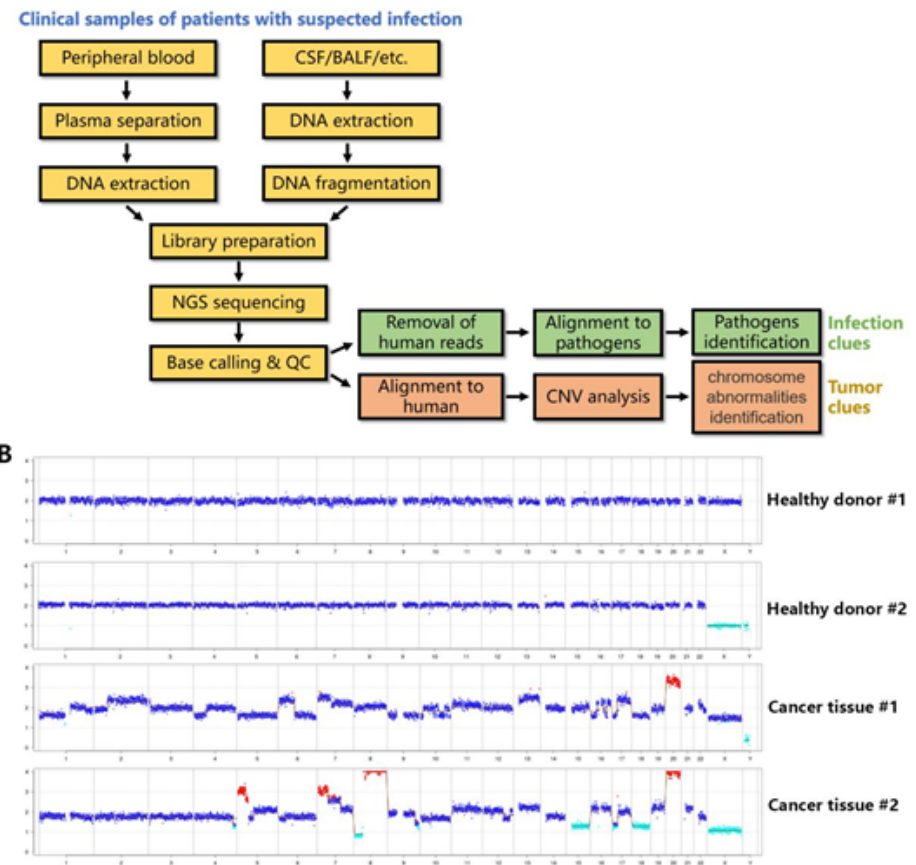

Figure 2

Integration of CNV analysis with mNGS to detect tumors. a Onco-mNGS workflow. b CNV data derived from two healthy donors and two patients with colorectal cancer. Abbreviations: CSF: cerebrospinal fluid; BALF: bronchoalveolar lavage fluid; CNV: copy number variation.

A

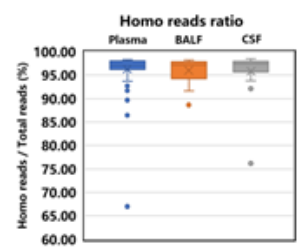

C

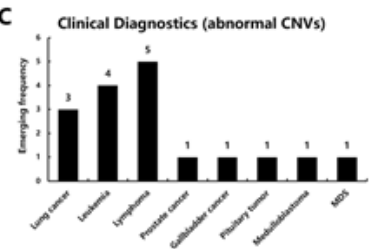

B

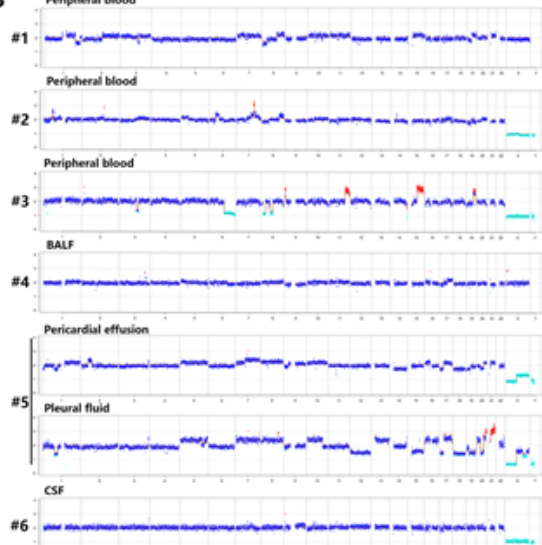

E
D

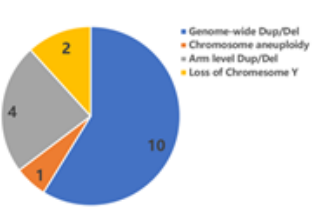

Onco-mNGS analysis $\quad$ Clues to malignancy Total

\begin{tabular}{rcccc}
\multicolumn{2}{c}{ Onco-mNGS analysis } & Postive & Negative & \multicolumn{1}{c}{ Total } \\
\cline { 2 - 5 } Clues to infection & Postive & $\mathbf{5}$ & $\mathbf{6 6}$ & $71(50.7 \%)$ \\
\cline { 2 - 5 } & Negative & $\mathbf{1 2}$ & $\mathbf{5 7}$ & $69(49.3 \%)$ \\
\hline Total & $17(12.1 \%)$ & $123(87.9 \%)$ & 140 \\
\hline
\end{tabular}

Figure 3

CNV analysis of cancer patients. a The homo reads ratios of three sample types (peripheral blood, BALF and CSF). b Abnormal CNVs of patients 1-6 with confirmed malignant tumors who were not recognized before CNV analysis. Patient 6 , suspected of having central nerve system lymphoma, refused a biopsy. c The clinical diagnosis of 17 patients with abnormal CNVs. d Classification of chromosome abnormalities in 17 patients. e Summarization of the NGS results of 140 patients with infection and tumor clues. 
A

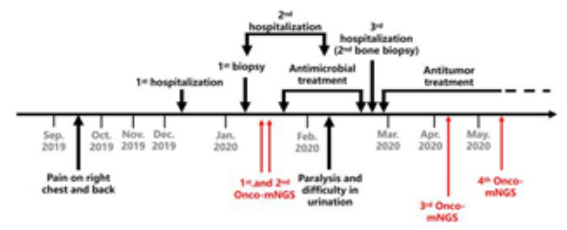

C

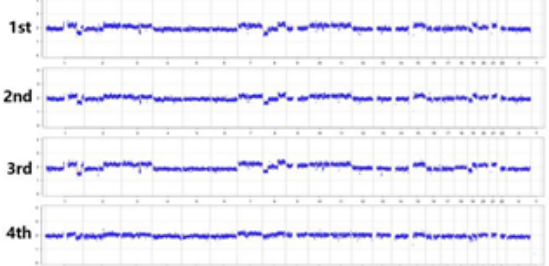

B

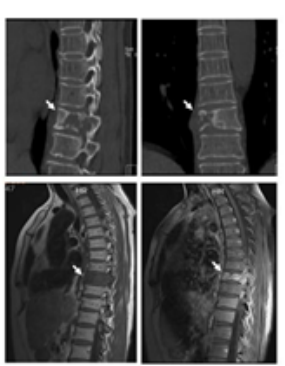

D

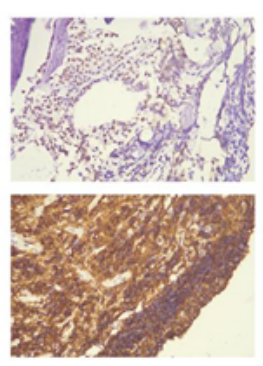

\section{Figure 4}

Schematic diagram of Onco-mNGS and a clinical case in which Onco-mNGS was applied and cancer was diagnosed. a Timeline beginning with the patient's initial complaint and ending with a final diagnosis. Major events during the course of the patient's illness are indicated by arrows. b CT scans obtained during the patient's first hospitalization revealed bone destruction of the 9th thoracic vertebrae and right appendix with a paravertebral soft tissue shadow. The lesion is indicated by the white arrow. c Four CNV results generated at different sampling times. $d$ Results of the repeated bone biopsy showing infiltrating, actively proliferating $\mathrm{B}$ lymphocytes evidenced by $60 \%$ CD20 and Ki67 positivity.

\section{Supplementary Files}

This is a list of supplementary files associated with this preprint. Click to download.

- SupplementalmaterialsGM20200220.docx 